\title{
Maximal Function Estimates for the Parabolic Mean Value Kernel
}

\author{
Hugo AIMAR, Ivana GómEZ, \\ and Bibiana IAFFEI \\ Instituto de Matemática Aplicada del Litoral \\ Departamento de Matemática, FIQ$$
\text { CONICET - UNL }
$$ \\ Güemes 3450, S3000GLN Santa Fe - Argentina \\ haimar@santafe-conicet.gov.ar \\ Instituto de Matemática Aplicada del Litoral \\ Departamento de Matemática, FICH \\ CONICET - UNL \\ Güemes 3450, S3000GLN Santa Fe - Argentina \\ ivanagomez@santafe-conicet.gov.ar

\footnotetext{
Instituto de Matemática Aplicada del Litoral

Departamento de Matemática, FHUC

CONICET - UNL

biaffei@santafe-conicet.gov.ar
} \\ Güemes 3450, S3000GLN Santa Fe - Argentina
}

Received: August 1, 2007

Accepted: December 12, 2007

\section{ABSTRACT}

We obtain parabolic and one-sided maximal function estimates for nonisotropic dilations of the mean value kernel for the heat equation

$$
k(x, t)=\frac{1}{4} \frac{|x|^{2}}{t^{2}} \mathcal{X}_{E(0,0 ; 1)}(x,-t),
$$

where $E(0,0 ; 1)$ is the heat ball given by

$$
\left\{(x, t) \in \mathbb{R}^{d+1}: t \leq 0, \quad(-4 \pi t)^{\frac{d}{2}} e^{-\frac{|x|^{2}}{4 t}} \leq 1\right\}
$$

Key words: one-sided parabolic maximal function, heat equation, mean value formula. 2000 Mathematics Subject Classification: 42B25, 35K05.

The authors were supported by CONICET, CAI+D(UNL), and ANPCyT. 


\section{Introduction}

The basic mean value formula for temperatures in $\mathbb{R}^{d+1}$, i.e., for solutions of $\frac{\partial u}{\partial t}=\Delta u$, states that, if $u$ is a smooth solution of the heat equation in the domain $\Omega$ of $\mathbb{R}^{d+1}$, the identity

$$
u(x, t)=\iint_{\Omega} k_{\delta}(x-y, t-s) u(y, s) d y d s
$$

holds for every $(x, t) \in \Omega$ and every $\delta>0$ for which the support of $k_{\delta}(x-\cdot, t-\cdot)$ is contained in $\Omega$. Here $k_{\delta}$ denotes the parabolic mollifier of $k: k_{\delta}(x, t)=\frac{1}{\delta^{d+2}} k\left(\frac{x}{\delta}, \frac{t}{\delta^{2}}\right)$ for $\delta>0$. The kernel $k$ is given by $k(x, t)=\frac{1}{4} \frac{|x|^{2}}{t^{2}} \mathcal{X}_{E(0,0 ; 1)}(x,-t)$ where $E(0,0 ; 1)$ is the "heat ball" at the origin of space-time with "radius" one, given by

$$
E(0,0 ; 1)=\left\{(x, t) \in \mathbb{R}^{d+1}: t \leq 0,(-4 \pi t)^{\frac{d}{2}} e^{-\frac{|x|^{2}}{4 t}} \leq 1\right\}
$$

For a proof of this formula see, for example, [1, Theorem 3, page 52]. See also [7].

For $f \in L_{\mathrm{loc}}^{1}\left(\mathbb{R}^{d+1}\right)$ and $(x, t) \in \mathbb{R}^{d+1}$, set

$$
\left(k_{\delta} * f\right)(x, t)=\iint_{\mathbb{R}^{d+1}} k_{\delta}(x-y, t-s) f(y, s) d y d s .
$$

In this note we aim to get estimates for the maximal operator $k^{*} f=\sup _{\delta>0}\left|k_{\delta} * f\right|$ in terms of Hardy-Littlewood type operators which preserve, as much a possible, the two basic features of the kernels $k_{\delta}$ :

(i) the parabolic shape of the supports,

(ii) the one-sided behavior of $k_{\delta}$ as functions of $t$.

To state the result of this note let us start by introducing some well-known maximal operators. By $M_{n}$ we shall denote the centered Hardy-Littlewood maximal function defined by the Euclidean balls on $\mathbb{R}^{n}$, in other words,

$$
M_{n} f(z)=\sup _{r>0} \frac{1}{|B(z ; r)|} \int_{B(z ; r)}|f(\zeta)| d \zeta .
$$

The left one-sided maximal function is defined, on a locally integrable function $g$ of a real variable $t$, by

$$
M_{1}^{-} g(t)=\sup _{h>0} \frac{1}{h} \int_{t-h}^{t}|g(s)| d s .
$$

The parabolic Hardy-Littlewood maximal function is defined by

$$
\mathcal{M} f(x, t)=\sup _{r>0} \frac{1}{|\mathcal{B}(x, t ; r)|} \iint_{\mathcal{B}(x, t ; r)}|f(y, s)| d y d s
$$


where $\mathcal{B}(x, t ; r)=\left\{(y, s) \in \mathbb{R}^{d+1}:\left(\frac{|x-y|}{r}\right)^{2}+\left(\frac{|t-s|}{r^{2}}\right)^{2}<1\right\}$. With $\mathcal{M}^{-} f(x, t)$ we denote the parabolic maximal operator with respect to the "lower halves" of the parabolic balls

$$
\mathcal{M}^{-} f(x, t)=\sup _{r>0} \frac{1}{\left|\mathcal{B}^{-}(x, t ; r)\right|} \iint_{\mathcal{B}^{-}(x, t ; r)}|f(y, s)| d y d s
$$

where $\mathcal{B}^{-}(x, t ; r)=\{(y, s) \in \mathcal{B}(x, t ; r): s \leq t\}$.

The following statement contains the pointwise estimates for $k^{*}$ that we shall prove in the next sections.

Main theorem. The following estimates hold true for every $(x, t) \in \mathbb{R}^{d+1}$, and some dimensional constant $C$ :

$$
\begin{aligned}
& k^{*} f(x, t) \leq C \mathcal{M} f(x, t), \\
& k^{*} f(x, t) \leq C M_{1}^{-} M_{d} f(x, t),
\end{aligned}
$$

where the right hand side is the iterated maximal operator obtained by the composition of $M_{d}$ acting on the space variable, for fixed time, and $M_{1}^{-}$, the one-sided one dimensional maximal operator on the time variable, explicitly

$$
M_{1}^{-} M_{d} f(x, t)=\sup _{h>0} \frac{1}{h} \int_{t-h}^{t}\left(\sup _{r>0} \frac{1}{r^{d}} \int_{B(x ; r)}|f(y, s)| d y\right) d s .
$$

Moreover,

$$
k^{*} f(x, t) \leq C \mathcal{M}^{-} f(x, t) .
$$

Let us notice that the inequalities

$$
\mathcal{M}^{-} f(x, t) \leq 2 \mathcal{M} f(x, t) \quad \text { and } \quad \mathcal{M}^{-} f(x, t) \leq M_{1}^{-} M_{d} f(x, t)
$$

are easily deduced from the very definition of all those maximal functions and the fact that fixed time sections of $\rho$-balls are Euclidean balls in $\mathbb{R}^{d}$. Hence to prove the main theorem we only have to show that (3) holds.

Regarding the meaning and consequences of (1) and (2), let us point out that the first one gives the weak type $(1,1)$ of $k^{*}$ and, as a by-product, a differentiation theorem for the family $k_{\delta}$ as $\delta \rightarrow 0$ for any $f \in L_{\text {loc }}^{1}\left(\mathbb{R}^{d+1}\right)$. Also (1) shows that the Muckenhoupt class $A_{p}(\mathcal{B})$ defined through the parabolic balls $\mathcal{B}$ provide good weights $w(x, t)$ for the $L^{p}\left(\mathbb{R}^{d+1}, w d x d t\right)$ boundedness of $k^{*}, 1 \leq p<\infty$. Inequality (2), with a right hand side given by iteration of two maximal operators, do not give such a good information about the behavior of $k^{*}$ on $L^{1}$ spaces, it gives instead another information for the $L^{p}\left(\mathbb{R}^{d+1}, w d x d t\right)$ boundedness of $k^{*}$ when $p>1$. In fact, from the results of $[4,5]$, weights which do not belong to $A_{p}(\mathcal{B})$ still satisfy the inequality

$$
\left\|k^{*} f\right\|_{L^{p}\left(\mathbb{R}^{d+1}, w d x d t\right)} \leq C\|f\|_{L^{p}\left(\mathbb{R}^{d+1}, w d x d t\right)} .
$$


For some one-sided weight theory for dimension larger than 1 see [2]. Let us also observe that, since for continuous functions $f$ defined on $\mathbb{R}^{d+1}$ we have that $k_{\delta} * f(x)$ converges to $f(x)$ at every point $x$, hence these $L^{p}$ type estimates for $k^{*}$ imply that $\left\{k_{\delta} * f: \delta>0\right\}$ is a good approximation of the identity on each one of those Lebesgue spaces.

For the proof of (3) we shall use a parabolic extension of the well-known criteria of the "integrable radial upper estimate" for the kernel (see $[3,6]$ ) that is satisfied by the mean value kernel $k$ for every $d \geq 1$. The above mentioned extension, which could also be obtained for more general parabolic dilations or for some classes of spaces of homogeneous type, is given in Theorem 1.1. The main point of this note is the proof of the main theorem, which is contained in section 2 .

Notice that since in the definition of $k^{*}$ we are dealing with parabolic dilations instead of Euclidean ones, we do not have an estimate for $k^{*}$ in terms of $M_{d+1}$. Moreover, even when the parabolic mean value kernel $k(x, t)$ admits an integrable, "radial-parabolic" majorant, for $d=1, k(x, t)$ does not admit an integrable, "radialelliptic" majorant. Lemma 2.1 contains this result.

\section{One-sided parabolic approximate identities}

For $x \in \mathbb{R}^{d}$ and $t \in \mathbb{R}$ with $|x|^{2}+t^{2}>0$, the unique positive solution of the equation

$$
\frac{|x|^{2}}{\rho^{2}}+\frac{t^{2}}{\rho^{4}}=1,
$$

denoted by $\rho(x, t)$ defines by $\rho(x-y, t-s)$ a distance on $\mathbb{R}^{d+1}$. Since the $\rho$-ball $\mathcal{B}(0,0 ; r)$ is the ellipsoid of $\mathbb{R}^{d+1}$ centered at the origin with semidiameters $r, \ldots, r, r^{2}$, we have that the $d+1$ dimensional Lebesgue measure of $\mathcal{B}(0,0 ; r)$ is given by $c r^{d+2}$ with a constant $c$ which depends only on $d$. In fact, $\mathcal{B}(0,0 ; r)$ is given by $T_{r}(\mathcal{B}(0,0 ; 1))$ with $T_{r}$ the parabolic dilation $T_{r}(y, s)=\left(r y, r^{2} s\right)$. Moreover, since the unit ball for the distance $\rho$ is the same as the Euclidean unit ball $B(0,0 ; 1)$, we have $\mathcal{B}(0,0 ; r)=$ $T_{r}(B(0,0 ; 1))$.

Given a real measurable function $K$ defined on $\mathbb{R}^{d+1}$ and a positive real number $\delta$, set $K_{\delta}(x, t)=\frac{1}{\delta^{d+2}} K\left(\frac{x}{\delta}, \frac{t}{\delta^{2}}\right)$. Let us consider the following univariate nonincreasing function defined on $\mathbb{R}^{+}$by

$$
\kappa(\lambda)=\sup _{\rho(x, t) \geq \lambda}|K(x, t)|
$$

With the above definitions we are in position to state and prove the result of this section.

Theorem 1.1. Let $K$ be a measurable kernel on $\mathbb{R}^{d+1}$ which vanishes for $t<0$. Assume that for $\rho$ given by (4) and $\kappa$ given by (5), the function $\kappa \circ \rho(x, t)=\kappa(\rho(x, t))$ 
is in $L^{1}\left(\mathbb{R}^{d+1}\right)$. Then there exists a constant $C$, depending only on $d$ and the $L^{1}$ norm of $\kappa \circ \rho$, such that the inequality

$$
K^{*} f(x, t) \leq C \mathcal{M}^{-} f(x, t)
$$

holds for every $(x, t) \in \mathbb{R}^{d+1}$, where $K^{*} f(x, t)=\sup _{\delta>0}\left|\left(K_{\delta} * f\right)(x, t)\right|$.

Proof. For fixed $\delta>0$ and $(x, t) \in \mathbb{R}^{d+1}$, we have

$$
\begin{aligned}
\left|\left(K_{\delta} * f\right)(x, t)\right| & \leq \iint_{\{s \geq 0\}}\left|K_{\delta}(y, s)\right||f(x-y, t-s)| d y d s \\
& =\frac{1}{\delta^{d+2}} \iint_{\{s \geq 0\}}\left|K\left(\frac{y}{\delta}, \frac{s}{\delta^{2}}\right)\right||f(x-y, t-s)| d y d s \\
& \leq \frac{1}{\delta^{d+2}} \iint_{\{s \geq 0\}} \kappa\left(\rho\left(T_{\frac{1}{\delta}}(y, s)\right)\right)|f(x-y, t-s)| d y d s \\
& =\frac{1}{\delta^{d+2}} \iint_{\{s \geq 0\}} \kappa\left(\frac{\rho(y, s)}{\delta}\right)|f(x-y, t-s)| d y d s
\end{aligned}
$$

As usual, but with the parabolic distance instead of the Euclidean one, we proceed to decompose the domain of integration in a sequence of dyadic annuli with respect to $\rho$. Since $\kappa$ is nonincreasing and from the above remarks concerning the measure of $\rho$-balls we have

$$
\begin{aligned}
\left|\left(K_{\delta} * f\right)(x, t)\right| & \leq \frac{1}{\delta^{d+2}} \sum_{j \in \mathbb{Z}} \kappa\left(2^{j}\right) \quad \iint_{\left\{(y, s): s \geq 0, \delta 2^{j} \leq \rho(y, s)<\delta 2^{j+1}\right\}}|f(x-y, t-s)| d y d s \\
& \leq\left(\frac{1}{\delta^{d+2}} \sum_{j \in \mathbb{Z}}\left|\mathcal{B}^{-}\left(0,0 ; \delta 2^{j+1}\right)\right| \kappa\left(2^{j}\right)\right) \mathcal{M}^{-} f(x, t) \\
& =c\left(\sum_{j \in \mathbb{Z}} 2^{(d+2)(j+1)} \kappa\left(2^{j}\right)\right) \mathcal{M}^{-} f(x, t) .
\end{aligned}
$$

We only have to check that the series converges. But, from the monotonicity of $\kappa$ we can go backwards from the series to the integral

$$
\iint_{\mathbb{R}^{d+1}} \kappa(\rho(y, s)) d y d s .
$$

We would like to observe that the above result can be extended, with the same method, to much more general dilations than the $T_{r}$ used here (see for example [3]).

\section{Proof of the main theorem}

We shall show that the kernel

$$
k(x, t)=\frac{1}{4} \frac{|x|^{2}}{t^{2}} \mathcal{X}_{E(0,0 ; 1)}(x,-t)
$$


satisfies the hypotheses of Theorem 1.1. From the definition of the set $E(0,0 ; 1)$ we readily have that $k$ vanishes for $t<0$. Let us get a convenient upper estimate for

$$
\kappa(\lambda)=\sup _{\rho(x, t) \geq \lambda} k(x, t), \quad \lambda>0 .
$$

Set $E^{*}(0,0 ; 1)$ to denote the reflection of $E(0,0 ; 1)$ with respect to the hyperplane $\{t=0\}$. In other words $E^{*}(0,0 ; 1)=-E(0,0 ; 1)$. Notice first that if $\lambda>\frac{1}{\sqrt{4 \pi}}$, then the set $\left\{(x, t) \in \mathbb{R}^{d+1}: \rho(x, t) \geq \lambda\right\}$ does not intersect the support of $k$. Hence we can take $\kappa(\lambda)=0$ for $\lambda>\frac{1}{\sqrt{4 \pi}}$. On the other hand, for every $\lambda_{0}>0$ the kernel $\kappa(\lambda)$ is bounded above on the half line $\lambda \geq \lambda_{0}$ by a constant which, of course, depends on $\lambda_{0}$. Hence we only have to take care of the behavior of $\kappa$ for $\lambda$ small enough. Moreover it will be enough to bound $\sup _{\lambda_{0}>\rho(x, t) \geq \lambda} k(x, t)$ for some small $\lambda_{0}$ and every $\lambda \in\left(0, \lambda_{0}\right)$. For the intersection of the boundaries of $E^{*}(0,0 ; 1)$ and of $\mathcal{B}(0,0 ; \lambda)$ we have that

$$
\partial E^{*}(0,0 ; 1) \cap \partial \mathcal{B}(0,0 ; \lambda)=S(0, r(\lambda)) \times\{t(\lambda)\}
$$

for some positive numbers $r(\lambda)$ and $t(\lambda)$, where $S(0, r)$ is the $d-1$ dimensional Euclidean spherical surface centered at the origin of $\mathbb{R}^{d}$ with radius $r$. In fact, since the equation for $\partial E^{*}(0,0 ; 1)$ is given by

$$
|x|^{2}=2 d t \ln \frac{1}{4 \pi t}
$$

and $\partial \mathcal{B}(0,0 ; \lambda)$ is implicitly defined by

$$
\frac{|x|^{2}}{\lambda^{2}}+\frac{t^{2}}{\lambda^{4}}=1
$$

by substitution of (7) into (8) we have an equation in $t$ and $\lambda$ which for $\lambda$ small has one and only one solution $t(\lambda)>0$. Hence (6) holds with $r^{2}(\lambda)=2 d t(\lambda) \ln \frac{1}{4 \pi t(\lambda)}$. Notice now that, from the definition of $k(x, t)$, we have that for every $\left(x, t_{1}\right)$ and $\left(x, t_{2}\right)$ interior points of the set $E^{*}(0,0 ; 1)$ with $t_{1}<t_{2}, k\left(x, t_{1}\right) \geq k\left(x, t_{2}\right)$. Also if $(x, t)$ and $(y, t)$ belong to $E^{*}(0,0 ; 1)$ and $|x| \leq|y|$ then $k(x, t) \leq k(y, t)$. Next let us take a look of the restriction of $k$ to the boundary of $E^{*}(0,0 ; 1)$. Taking into account $(7)$, we have that $\left.k\right|_{\partial E^{*}}$ is given, as a function of $t>0$, by

$$
\frac{d}{2 t} \ln \frac{1}{4 \pi t},
$$

which is certainly decreasing as a function of $t$. The above remarks show that the supremum of $k(x, t)$ inside the parabolic annulus $\lambda_{0}>\rho(x, t) \geq \lambda$ is attained at any point of the spherical surface $S(0, r(\lambda)) \times\{t(\lambda)\}$, and, from (9),

$$
\sup _{\lambda_{0}>\rho(x, t) \geq \lambda} k(x, t)=\frac{d}{2 t(\lambda)} \ln \frac{1}{4 \pi t(\lambda)} .
$$


By substitution of (7) in (8) we have a quadratic expression in $\frac{t}{\lambda^{2}}$ given by

$$
\left(\frac{t}{\lambda^{2}}\right)^{2}+2 d \ln \frac{1}{4 \pi t}\left(\frac{t}{\lambda^{2}}\right)-1=0 .
$$

Hence, since $t>0$, we have the following relation for $t$ and $\lambda$

$$
\frac{t}{\lambda^{2}}=-d \ln \frac{1}{4 \pi t}+\sqrt{d^{2} \ln ^{2} \frac{1}{4 \pi t}+1}
$$

from which $\lambda(t)=\frac{\sqrt{t}}{a(t)}$ where

$$
a(t)=\sqrt{\sqrt{d^{2} \ln ^{2} \frac{1}{4 \pi t}+1}-d \ln \frac{1}{4 \pi t}} .
$$

The following properties of the function $\lambda(t)$ are easy to check and they provide a lower bound for $t(\lambda)$ for $\lambda$ small enough, leading us to get an integrable upper bound for $\kappa \circ \rho$,

(i) for $t>0$ small enough, $\lambda^{\prime}(t)>0$;

(ii) for every $\varepsilon \in\left(0, \frac{1}{2}\right)$ there exists $t_{0}(\varepsilon)>0$ such that $\lambda(t) \leq t^{\frac{1}{2}-\varepsilon}$ for every $t \in\left(0, t_{0}(\varepsilon)\right)$

Let us first show how the main theorem follows from (i) and (ii). From (i) the inequality in (ii) can be rewritten in terms of inverse functions as

$$
t(\lambda) \geq \lambda^{\frac{2}{1-2 \varepsilon}}
$$

for $0<\lambda<\lambda\left(t_{0}(\varepsilon)\right)$. By substitution of inequality above in (10), for those values of $\lambda$, we get the estimate

$$
\begin{aligned}
\sup _{\lambda\left(t_{0}(\varepsilon)\right)>\rho(x, t) \geq \lambda} k(x, t) & \leq \frac{d}{2 \lambda^{\frac{2}{1-2 \varepsilon}}} \ln \frac{1}{4 \pi \lambda^{\frac{2}{1-2 \varepsilon}}} \\
& \leq \frac{d}{1-2 \varepsilon} \lambda^{\varepsilon} \ln \left(\frac{(4 \pi)^{\frac{2 \varepsilon-1}{2}}}{\lambda}\right) \frac{1}{\lambda^{\frac{2}{1-2 \varepsilon}+\varepsilon}} \\
& \leq \frac{c}{\lambda^{\beta}}
\end{aligned}
$$

where $\beta=\frac{2}{1-2 \varepsilon}+\varepsilon$. Taking $\varepsilon=\frac{1}{8}$ and $\lambda_{0}=\lambda\left(t_{0}\left(\frac{1}{8}\right)\right)$, we have that $\beta<3 \leq d+2$ for $d=1,2,3, \ldots$ Hence for every $(x, t) \in \mathcal{B}\left(0,0 ; \lambda_{0}\right)$ we have that

$$
\kappa(\rho(x, t)) \leq \frac{c}{\rho^{\beta}(x, t)}
$$


with $\beta<d+2$. Now by dyadic decomposition of the $\rho$-ball centered at $(0,0)$ with radius $\lambda_{0}$, we get

$$
\begin{aligned}
\iint_{\mathcal{B}\left(0,0 ; \lambda_{0}\right)} \frac{d x d t}{\rho^{\beta}(x, t)} & =\sum_{j=0}^{\infty} \iint_{\left\{(x, t): \lambda_{0} 2^{-(j+1)} \leq \rho(x, t)<\lambda_{0} 2^{-j}\right\}} \frac{d x d t}{\rho^{\beta}(x, t)} \\
& \leq \lambda_{0}^{-\beta} \sum_{j=0}^{\infty}\left|\mathcal{B}\left(0,0 ; \lambda_{0} 2^{-j}\right)\right| 2^{\beta(j+1)} \\
& =c\left(\lambda_{0}, d\right) \sum_{j=0}^{\infty} 2^{-(d+2-\beta) j}<\infty .
\end{aligned}
$$

Hence, from these estimates and the remark at the beginning of the proof we get that $\kappa \circ \rho \in L^{1}\left(\mathbb{R}^{d+1}\right)$ and the Theorem 1.1 can be applied.

Let us finally sketch the proofs of (i) and (ii). Setting $s=d \ln \frac{1}{4 \pi t}$, to prove (i), we only have to check that $\Lambda^{\prime}(s)<0$ for $s$ large enough where $\Lambda(s)$ is the positive solution of $4 \pi \Lambda^{2}(s)=e^{-\frac{s}{d}} /\left(\sqrt{s^{2}+1}-s\right)$. Which, in turn, is equivalent to show that the derivative of the last expression is negative for $s$ large. This derivative leads immediately to the inequality $1+\frac{s}{d}-\frac{1}{d} \sqrt{s^{2}+1}-\frac{s}{\sqrt{s^{2}+1}}<0$, which holds for $s \geq s_{0}$ for some $s_{0}$. In order to prove (ii), since $\lambda(t)=\frac{\sqrt{t}}{a(t)}=\Lambda(s)=e^{-\frac{s}{2 d}} /(\sqrt{4 \pi} A(s))$, with $A^{2}(s)=\sqrt{s^{2}+1}-s$, we only need to prove that for $s$ large enough $A^{2}(s) \geq$ $\frac{1}{(4 \pi)^{2 \varepsilon}} e^{-\frac{2 s}{d} \varepsilon}$. But the last inequality is equivalent to

$$
\ln \frac{\sqrt{s^{2}+1}+s}{(4 \pi)^{2 \varepsilon}} \leq \frac{2 \varepsilon}{d} s,
$$

which is certainly true for $s \geq s_{0}(\varepsilon, d)$. This finishes the proof of the main theorem.

Let us finally show that for $d=1$ the minimal "radial-elliptic" majorant does not belong to $L^{1}\left(\mathbb{R}^{2}\right)$.

Lemma 2.1. For $d=1$, in a small neighborhood of the origin of $\mathbb{R}^{2}$ the inequality

$$
\sup _{|(y, s)| \geq|(x, t)|} k(y, s) \geq \frac{c}{|(x, t)|^{2}}
$$

holds for some positive constant $c$.

Proof. We have to prove that $\bar{\kappa}(r)=\sup _{|(y, s)| \geq r} k(y, s) \geq \frac{c}{r^{2}}$ for $r>0$ small enough. To get a lower bound for $k(y, s)$ with $|(y, s)| \geq r$, we proceed as we did in the proof of the main theorem, substituting equation (8) by the Euclidean spherical surface in $\mathbb{R}^{d+1}$ of radius $r,|x|^{2}+t^{2}=r^{2}$. In this way, for the points in the intersection of the boundaries of $E^{*}(0,0 ; 1)$ and of $B(0,0 ; r)$, we get

$$
t^{2}+\left(2 d \ln \frac{1}{4 \pi t}\right) t=r^{2}(t)
$$


Notice that $\bar{\kappa}(r) \geq \frac{1}{4} \frac{|x|^{2}}{t^{2}}=\frac{1}{4}\left(\frac{r^{2}(t)}{t^{2}}-1\right)$ for $(x, t)$ in the set $\partial E^{*}(0,0 ; 1) \cap \partial B(0,0 ; r)$. Hence $\bar{\kappa}(r) \geq \frac{d}{2 t} \ln \frac{1}{4 \pi t}$, for $r=r(t)$. Since for $t$ small the function $\left(t+2 d \ln \frac{1}{4 \pi t}\right) \ln \frac{1}{4 \pi t}$ is bounded below by a positive constant, we have that $\bar{\kappa}(r) \geq \frac{c}{r^{2}}$ for some positive $c$.

\section{References}

[1] L. C. Evans, Partial differential equations, Graduate Studies in Mathematics, vol. 19, American Mathematical Society, Providence, RI, 1998.

[2] L. Forzani, F. J. Martín-Reyes, and S. Ombrosi, Weighted inequalities for the two-dimensional one-sided Hardy-Littlewood maximal function, preprint.

[3] M. de Guzmán, Real variable methods in Fourier analysis, North-Holland Mathematics Studies, vol. 46, North-Holland Publishing Co., Amsterdam, 1981. Notas de Matemática [Mathematical Notes], 75 .

[4] F. J. Martín-Reyes, New proofs of weighted inequalities for the one-sided Hardy-Littlewood maximal functions, Proc. Amer. Math. Soc. 117 (1993), no. 3, 691-698.

[5] E. Sawyer, Weighted inequalities for the one-sided Hardy-Littlewood maximal functions, Trans. Amer. Math. Soc. 297 (1986), no. 1, 53-61.

[6] E. M. Stein, Singular integrals and differentiability properties of functions, Princeton Mathematical Series, vol. 30, Princeton University Press, Princeton, N.J., 1970.

[7] N. A. Watson, A theory of subtemperatures in several variables, Proc. London Math. Soc. (3) 26 (1973), 385-417. 